\section{Serum Neopterin as a Novel Marker for Carotid Artery Stenosis in Community Subjects}

\author{
Huanquan Liao', Hua Hong ${ }^{1 *}$ and Hongxuan Wang ${ }^{2}$ \\ ${ }^{1}$ Department of Neurology, The First Affiliated Hospital, Sun Yat-Sen \\ University, Guangzhou, Guangdong Province, China \\ ${ }^{2}$ Department of Neurology, The Second Affiliated Hospital, Sun Yat-Sen \\ University, Guangzhou, Guangdong Province, China
}

\begin{abstract}
Background: Carotid Artery Stenosis (CAS) is one of the major high-risk mechanisms of stroke. Neopterin, an immune modulator secreted by macrophages, have been reported to be elevated in patients with cerebrovascular disease. However, an association between serum neopterin level and CAS has not been firmly established.

Objective: To investigate the cross-sectional relationship between serum neopterin levels and CAS assessed by ultrasound.

Methods: The study participants were volunteers in a community-based cohort aiming to prevent stroke in Guangzhou. Bilateral carotid artery ultrasonography was performed to assess the carotid atherosclerosis. After participant criterion screening and random sampling, finally 140 subjects were studied in our present study. Enzyme-linked immunosorbent assay was performed to measured serum neopterin concentrations.

Results: Neopterin level in the CAS and control group was 5.4 (3.3-7.3) $\mathrm{nmol} / \mathrm{L}$ and $4.6(3.6-5.4) \mathrm{nmol} / \mathrm{L}$ respectively, with a significant difference $(P<0.05)$. After multivariate adjusted, neopterin levels remained an independent factor. With per $1.0 \mathrm{nmol} / \mathrm{L}$ (approximately $1 \mathrm{IQR})$ increased, the OR value was $1.84(95 \% \mathrm{Cl}$ : 1.25-2.71, $\mathrm{P}<0.05$ ). ROC curve of serum neopterin concentrations for the prediction of CAS revealed that the area under curve was $0.665(P<0.01)$. The cut-off value of $5.1 \mathrm{nmol} / \mathrm{L}$ had a sensitivity of $68.6 \%$ and specificity of $61.5 \%$ in detecting CAS.

Conclusion: These findings demonstrate that circulating neopterin level is increased in subclinical carotid atherosclerosis population with CAS and reinforce the key roles of inflammatory response in the pathogenesis.
\end{abstract}

*Corresponding author: Hua Hong, Department of Neurology, The First Affiliated Hospital, Sun Yat-Sen University, 58 Zhongshan Road 2, Guangzhou 510080, Guangdong Province, China, Tel: +86 15920500906; Fax: +86 2087331989; E-mail: sumsdiy@163.com

Citation: Liao H, Hong H, Wang H (2015) Serum Neopterin as a Novel Marke for Carotid Artery Stenosis in Community Subjects. J Cardiol Stud Res 2: 009.

Received: September 17, 2015; Accepted: October 19, 2015; Published: November 02, 2015

Copyright: (C) 2015 Liao $\mathrm{H}$, et al. This is an open-access article distributed under the terms of the Creative Commons Attribution License, which permits unrestricted use, distribution, and reproduction in any medium, provided the original author and source are credited.
Keywords: Carotid; Carotid artery stenosis; Neopterin; Ultrasound

$\begin{array}{ll}\text { Abbreviations } \\ \text { AUC - } & \text { Area Under Curve } \\ \text { BMI - } & \text { Body Mass Index } \\ \text { CAA - } & \text { Carotid Artery Atherosclerosis } \\ \text { CAS - } & \text { Carotid Artery Stenosis } \\ \text { DBP - } & \text { Diastolic Blood Pressure } \\ \text { EDV - } & \text { End Diastolic Velocity } \\ \text { ELISA - } & \text { Enzyme-Linked Immunosorbent Assay } \\ \text { FBG - } & \text { Fasting Blood Glucose } \\ \text { HDL-C - } & \text { High Density Lipoprotein-Cholesterol } \\ \text { hs-CRP - high-sensitivity C-Reaction Protein } \\ \text { ICA - } & \text { Internal Carotid Artery } \\ \text { IMT - } & \text { Intima-Media Thickness } \\ \text { IQR - } & \text { Interquartile Range } \\ \text { LDL-C - } & \text { Low Density Lipoprotein Cholesterol } \\ \text { OR - } & \text { Odds Ratio } \\ \text { PSV - } & \text { Peak Systolic Velocity } \\ \text { ROC - } & \text { Receiver Operating Characteristic } \\ \text { SBP - } & \text { Systolic Blood Pressure } \\ \text { SD - } & \text { Standard Deviation } \\ \text { TC - } & \text { Total Cholesterol } \\ \text { TG - } & \text { Triglyceride } \\ \text { UA - } & \text { Uric Acid } \\ \text { WHR - } & \text { Waist Hip Ratio } \\ \end{array}$

\section{Introduction}

Stroke remains one of the most devastating neurological diseases, often causing death, or gross physical impairment [1]. Carotid Artery Atherosclerosis (CAA) is one of the major high-risk mechanisms of stroke [2]. Epidemiologic estimates of first-time ischemic stroke attributable to CAA vary, but range from roughly 7 to $18 \%$ of all incident stroke $[3,4]$. Cerebral infarction may result from the reduction in cerebral perfusion pressure that occurs distal to a tight Carotid Artery Stenosis (CAS) or occlusion [5,6]. While an embolism may also contribute, the association of border zone infarcts with CAS emphasizes the significance of hemodynamic disturbance in the pathogenesis of these types of infarct [6]. Numerous publications from recent years indicate a significant contribution of inflammatory factors to the development and progression of atherosclerosis [7]. Atherosclerotic lesions in the carotid arteries have been associated with increased serum levels of different inflammatory markers, such as C-reactive protein and fibrinogen [8,9]. Inflammatory cytokines enter the circulation in soluble form. The concept of the involvement of inflammation in atherosclerosis has validated the adoption of inflammatory biomarkers for cerebrovascular risk stratification and prediction [10]. Neopterin is a pteridine derivative of the body fluids which is biologically stable, mainly produced by macrophages stimulated by $\gamma$-IFN which is secreted by activated $T$ cells lymphocytes [11], and is a specific marker for activated mononuclear macrophages [12]. In fact, elevated neopterin levels have been reported in patients with coronary artery disease compared with controls $[13,14]$. Astonishingly, an association 
between serum neopterin level and CAS has not been firmly established. In this context, we hypothesized that neopterin may be related to CAS. Thus, the current study sought to achieve this objective by measuring circulating serum neopterin levels in community subjects and investigating the cross-sectional relationship between serum neopterin levels and CAS assessed by ultrasound.

\section{Materials and Methods}

\section{Participant enrollment, screening and selection}

Study participants were residents living in Nonglin community in the center of Guangzhou, China. Residents were recruited in a community-based cohort aiming to prevent stroke by the organization of Department of Neurology, the First Affiliated Hospital, Sun Yat-Sen University from July 2008 to December 2008. Residents who were volunteers, aged from 46 to 75 , and willing to be re-examined in future were eligible. Residents were excluded if they lived in other districts, refused to complete necessary questionnaires and examinations in the study or had malignant tumors, acute myocardial infarction, and other critical illnesses which would possibly affect the follow-up in the future. Altogether 962 participants were recruited. The study protocol was approved by the Ethics Committee of the First Affiliated Hospital of Sun Yat-Sen University and all participants gave written informed consent of the study. In the present study, participants were further excluded if they had diseases which corroboratively or potentially affect the function of the immune system including cardiovascular disease, cerebrovascular disease, chronic renal failure, chronic liver insufficiency, blood diseases, asthma, acute infectious diseases, or autoimmune diseases. After the present study criterion screening, 534 participants were eligible for our present study. Among these participants, there were 183 (34.3\%) subjects with CAS, other 351 (65.7\%) subjects without CAS were taken as controls. Eventually, random sampling was used to select the sample for the study. Finally 70 participants were respectively selected from the CAS and control groups. Totally 140 subjects were studied in our present study.

\section{Medical and physical risk factors}

Demographic variables, histories of previous diseases, cigarette smoking, and alcohol consumption were obtained by well trained investigators by face to face interview in clinics using a standardized questionnaire. Cigarette smoking was defined as the subject had smoked more than 1 cigarette per day for more than half a year. Alcohol consumption was defined as the subject had drunk any kind of alcohol drinks more than once a week regularly for more than half a year. Previous histories of hypertension, diabetes, hyperlipidemia, heart disease or stroke were defined if the subject had been diagnosed with the disease in hospital before or had used medications regularly. Waist and hip circumference, weight and height were measured and then Waist Hip Ratio (WHR) was calculated. Body Mass Index (BMI) was calculated as weight $(\mathrm{kg})$ divided by the square of height $\left(\mathrm{m}^{2}\right)$. Blood pressure was measured with a mercury sphygmomanometer in the morning.

\section{Biological risk factors}

Fasting venous blood samples for Fasting Blood Glucose (FBG), Total Cholesterol (TC), Triglyceride (TG), Low Density Lipoprotein Cholesterol (LDL-C), High Density Lipoprotein Cholesterol (HDL-C), high-sensitivity C-Reaction Protein (hs-CRP), and Uric Acid (UA) were collected in the morning and then analyzed by the enzyme method in autoanalyzing machine. The coefficients of variance of repeated measurements of the chemistry tests were $1.0 \%$ to $1.6 \%$.

\section{Ultrasound assessment of CAS}

Carotid ultrasound measurements were performed with one $B$ mode ultrasound system, APLIO XU equipped with a 7.5 MHz linear array transducer (TOSHIBA, USA) by 2 experienced vascular sonographers. Subjects were examined in supine position with their necks extended. The three bilateral defined locations including common carotid (20 $\mathrm{mm}$ proximal to the bifurcation), carotid bifurcation and Internal Carotid Artery (ICA, $10 \mathrm{~mm}$ distal to bifurcation) were measured longitudinally and transversely. The scan and measurements were performed according to internationally recognized guidelines [15]. Intima-Media Thickness (IMT) was measured as the distance between the two parallel echogenic lines on the far wall of artery in longitudinal plane image frozen in the screen by electronic calipers [16]. Plaque was defined as localized thickening of IMT $\geq 1.3 \mathrm{~mm}$ which did not uniformly involve the whole wall of carotid artery [17]. Classification of ultrasound plaque appearance was performed independently from the videotape by 1 single operator blinded to all other data. 3 categories were classified as (1) no plaques; (2) echogenic plaques; (3) echolucent plaques. Echolucent plaques are mainly composed by the deposition of cholesterol, necrosis tissue and plaque bleeding, a type of plaque with instability [18]. The carotid stenosis measurements were performed according to internationally recognized guidelines [8]. The Peak Systolic Velocity (PSV) and End Diastolic Velocity (EDV) were measured in the common and internal carotid arteries, and the degree of stenosis was then calculated according to St. Mary's Ratio which is the ratio of the PSV in the internal carotid arteries, a value that increases with degree of stenosis, divided by the EDV in the distal common carotid artery, a value that decreases with increasing resistance in the internal carotid arteries due to stenosis (Table 1) [19]. Table 1, we had assessed interobserver reproducibility of CAS detection by repeated scans on several randomly selected participants. The kappa value for the agreement of CAS was $0.738(\mathrm{P}<0.01)$, which was acceptable.

\begin{tabular}{|c|c|}
\hline St. Mary's Ratio & ICA Stenosis (\%) \\
\hline$<8$ & $<50$ \\
\hline $8-10$ & $50-59$ \\
\hline $11-13$ & $60-69$ \\
\hline $14-21$ & $70-79$ \\
\hline $22-29$ & $80-89$ \\
\hline$>30$ & $>90$ but less than near occlusion \\
\hline Variable & Near occlusion \\
\hline \multicolumn{2}{|c|}{ Table 1: Calculation of St. Mary's Ratio. }
\end{tabular}

\section{Measurements of serum neopterin}

Circulating serum neopterin concentrations were measured using a commercially available solid phase Enzyme-Linked Immunosorbent Assay (ELISA) kit (IBL, Hamburg, Germany) based on the basic principle of a competitive ELISA. An unknown amount of antigen in the sample and a fixed amount of enzyme labelled antigen compete for the antibody-binding sites (rabbit-anti-neopterin). Both antigen-antibody complexes bind to the wells of the microtiter strips coated with a goat-anti-rabbit antibody. Unbound antigen is removed by washing. The intensity of the color developed after the substrate 
incubation is inversely proportional to the amount of antigen in the sample. Results of samples can be determined directly using the standard curve. In our study, inter-assay and intra-assay coefficients of variation were $<5.9 \%$ and $<8.9 \%$ respectively.

\section{Statistical analysis}

Analysis of normality of the continuous variables was performed with the Kolmogorov-Smirnov test. Results for continuous variables normally distributed were expressed as mean value \pm Standard Deviation (SD) and non-normally distributed (neopterin levels) was presented as median value (lower quartile-upper quartile). Dichotomized or categorized variables were described as numbers and proportions. In univariate analysis, continuous variables normally distributed were compared by student's t test. Continuous variable non-normally distributed was compared by nonparametric Mann-Whitney U test. Dichotomized or categorized variables were compared by Chi-square tests. The correlation between serum neopterin and hs-CRP levels was assessed using Pearson's coefficient correlation analysis. In order to adjust the confounding factors which is significant in univariate analysis, binary logistic regression analysis was performed to investigate the risk factors for CAS. Odds Ratio (OR) was calculated by logistic model. The Receiver Operating Characteristic (ROC) curve of the serum neopterin concentrations for predicting CAS and its Area Under Curve (AUC) analysis were performed. All statistic analysis was calculated with SPSS 13.0 software system (SPSS Inc., Chicago, IL, USA). A two-sided P-value of less than 0.05 was considered as statistically significant.

\section{Results}

\section{Baseline clinical characteristics and biochemical results}

In this research, totally 534 community subjects who had no diseases which corroboratively or potentially affected the function of the immune system including cardiovascular and cerebrovascular disease were eligible after screening of exclusion criterion, with an age of 57.1 \pm 5.6 years. The overall baseline clinical characteristics and biochemical results are shown in table 2. Carotid ultrasound revealed 183(34.3\%) subjects with CAS. In these subjects, most were mild stenosis $(<50 \%)$, only 7 subjects were moderate stenosis $(50-70 \%)$.

\section{Univariate comparison between CAS and control group}

Variables of risk factors of the participants in different groups are shown in table 3 . There were significant differences in age, WHR, BMI, history of hypertension, SBP, HDL-C and neopterin level $(\mathrm{P}<0.05)$. Neopterin level in the CAS and control group was 5.4 (3.3-7.3) $\mathrm{nmol} / \mathrm{L}$ and $4.6(3.6-5.4) \mathrm{nmol} / \mathrm{L}$ respectively (Figure 1$)$. However, hs-CRP level in the CAS and control group was $0.91(0.52-5.81) \mathrm{mg} / \mathrm{L}$ and $1.02(0.73-7.04) \mathrm{mg} / \mathrm{L}$ respectively, and the difference was not significant (Table 3).

\section{Comparison of neopterin levels in different plaque characteristics groups}

Neopterin level in the no plaque group, echogenic plaque group and echolucent plaque group were $4.6(2.8-7.8) \mathrm{nmol} / \mathrm{L}, 4.7$ (2.3-7.6) $\mathrm{nmol} / \mathrm{L}$ and $6.0(3.8-8.1) \mathrm{nmol} / \mathrm{L}$ respectively (Figure 2$)$. There were significant differences between no plaque group and echogenic plaque group, no plaque group and echolucent plaque group $(\mathrm{P}<0.05)$.

\begin{tabular}{|c|c|}
\hline Age (year) & $57.1 \pm 5.6$ \\
\hline Male, n (\%) & $158(29.6 \%)$ \\
\hline WHR & $0.88 \pm 0.06$ \\
\hline $\mathrm{BMI}\left(\mathrm{kg} / \mathrm{m}^{2}\right)$ & $23.6 \pm 3.3$ \\
\hline Smoking, n (\%) & $80(15.0 \%)$ \\
\hline Alcohol consumption, $\mathrm{n}(\%)$ & $30(5.6 \%)$ \\
\hline Hypertension, n (\%) & $125(23.4 \%)$ \\
\hline Diabetes, n (\%) & $44(8.2 \%)$ \\
\hline Hyperlipidemia, n (\%) & $141(26.4 \%)$ \\
\hline $\mathrm{SBP}(\mathrm{mmHg})$ & $127 \pm 17$ \\
\hline $\mathrm{DBP}(\mathrm{mmHg})$ & $79 \pm 10$ \\
\hline $\mathrm{TC}(\mathrm{mmol} / \mathrm{L})$ & $5.29 \pm 0.96$ \\
\hline TG $(\mathrm{mmol} / \mathrm{L})$ & $1.72 \pm 1.47$ \\
\hline HDL-C (mmol/L) & $1.38 \pm 0.32$ \\
\hline LDL-C (mmol/L) & $3.65 \pm 0.87$ \\
\hline $\mathrm{FBG}(\mathrm{mmol} / \mathrm{L})$ & $5.2 \pm 1.2$ \\
\hline $\mathrm{UA}(\mathrm{mmol} / \mathrm{L})$ & $295 \pm 51$ \\
\hline \multicolumn{2}{|c|}{ CAS } \\
\hline Yes, n (\%) & $183(34.3 \%)$ \\
\hline Mild & 176 \\
\hline Moderate & 7 \\
\hline no, n (\%) & $351(65.7 \%)$ \\
\hline
\end{tabular}

Table 2: Overall characteristics of 534 community participants.

\begin{tabular}{|c|c|c|c|}
\hline & CAS & Control & $\mathbf{P}$ \\
\hline $\mathrm{n},(\%)$ & $70(50.0 \%)$ & $70(50.0 \%)$ & \\
\hline Age (year) & $60.4 \pm 6.4$ & $55.7 \pm 5.5$ & $<0.01$ \\
\hline Male, $n(\%)$ & $24(34.3 \%)$ & $17(24.3 \%)$ & 0.19 \\
\hline WHR & $0.90 \pm 0.06$ & $0.86 \pm 0.06$ & $<0.01$ \\
\hline BMI $\left(\mathrm{kg} / \mathrm{m}^{2}\right)$ & $24.2 \pm 2.6$ & $22.4 \pm 3.2$ & $<0.01$ \\
\hline Smoking, n (\%) & $11(15.7 \%)$ & $4(5.7 \%)$ & 0.16 \\
\hline Alcohol consumption, $\mathrm{n}(\%)$ & $6(8.6 \%)$ & $4(5.7 \%)$ & 0.77 \\
\hline Hypertension, n (\%) & $26(37.1 \%)$ & $17(24.3 \%)$ & $<0.05$ \\
\hline Diabetes mellitus, n (\%) & $11(15.7 \%)$ & $4(5.7 \%)$ & 0.16 \\
\hline Hyperlipidemia, n (\%) & $24(34.3 \%)$ & $29(41.4 \%)$ & 0.57 \\
\hline $\mathrm{SBP}(\mathrm{mmHg})$ & $133 \pm 15$ & $124 \pm 18$ & $<0.01$ \\
\hline $\mathrm{DBP}(\mathrm{mmHg})$ & $80 \pm 9$ & $78 \pm 11$ & 0.13 \\
\hline $\mathrm{TC}(\mathrm{mmol} / \mathrm{L})$ & $5.34 \pm 0.91$ & $5.16 \pm 0.99$ & 0.26 \\
\hline $\mathrm{TG}(\mathrm{mmol} / \mathrm{L})$ & $1.70 \pm 0.81$ & $1.67 \pm 1.40$ & 0.88 \\
\hline $\mathrm{HDL}-\mathrm{C}(\mathrm{mmol} / \mathrm{L})$ & $1.32 \pm 0.28$ & $1.45 \pm 0.33$ & $<0.05$ \\
\hline LDL-C (mmol/L) & $3.79 \pm 0.84$ & $3.50 \pm 0.93$ & 0.06 \\
\hline $\mathrm{FBG}(\mathrm{mmol} / \mathrm{L})$ & $5.3 \pm 1.4$ & $5.1 \pm 0.8$ & 0.35 \\
\hline $\mathrm{UA}(\mathrm{mmol} / \mathrm{L})$ & $309 \pm 99$ & $284 \pm 82$ & 0.14 \\
\hline $\mathrm{hs}-\mathrm{CRP}(\mathrm{mg} / \mathrm{L})$ & $0.91(0.52-5.81)$ & $1.02(0.73-7.04)$ & 0.36 \\
\hline neopterin (nmol/L) & $5.4(3.3-7.3)$ & $4.6(3.6-5.4)$ & $<0.01$ \\
\hline
\end{tabular}

Table 3: Comparison between CAS and control group $(n=140)$.

\section{Correlation between serum neopterin and hs-CRP levels}

Figure 3, shows that there was a no significant correlation between serum neopterin and hs-CRP levels $(\mathrm{r}=0.121, \mathrm{P}=0.152)$. 


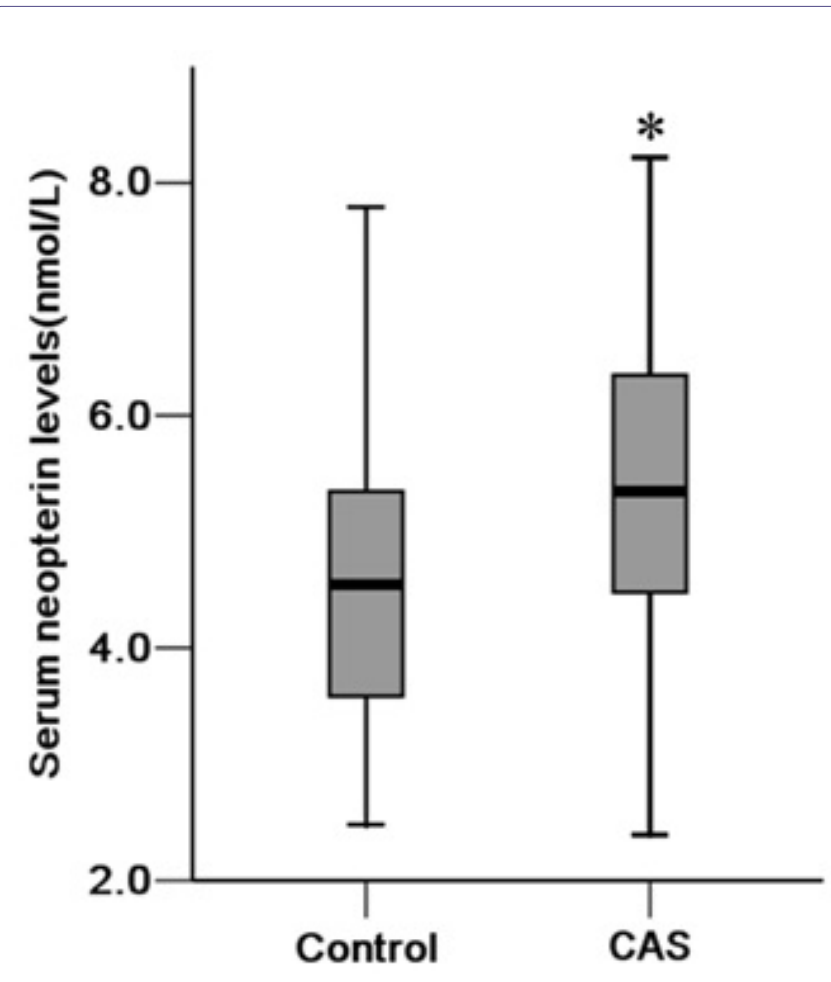

Figure 1: Comparison of neopterin levels between CAS and control groups.

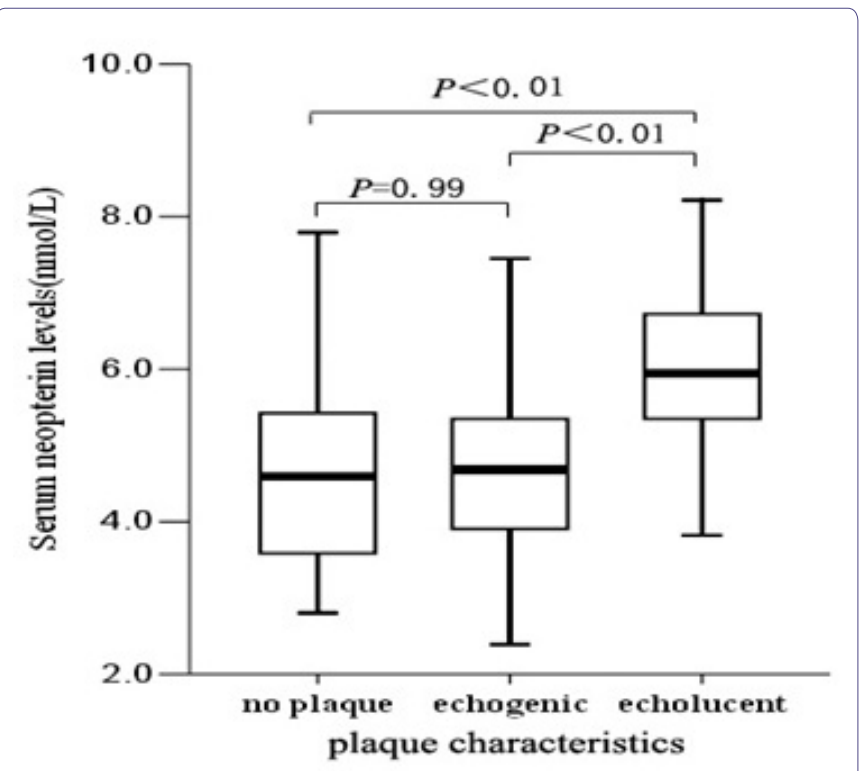

Figure 2: Comparison of neopterin levels in different plaque characteristics groups.

\section{Binary logistic regression analysis for the prediction of CAS}

In order to adjust the confounding factors which was significant in univariate analysis, binary logistic regression analysis was performed to investigate the risk factors for CAS (Table 4). After multivariate adjusted, neopterin levels remained an independent factor. With per $1.0 \mathrm{nmol} / \mathrm{L}$ (approximately $1 \mathrm{IQR}$ ) increased, the OR value was 1.84 (95\% CI: 1.25-2.71, $\mathrm{P}<0.05)$.

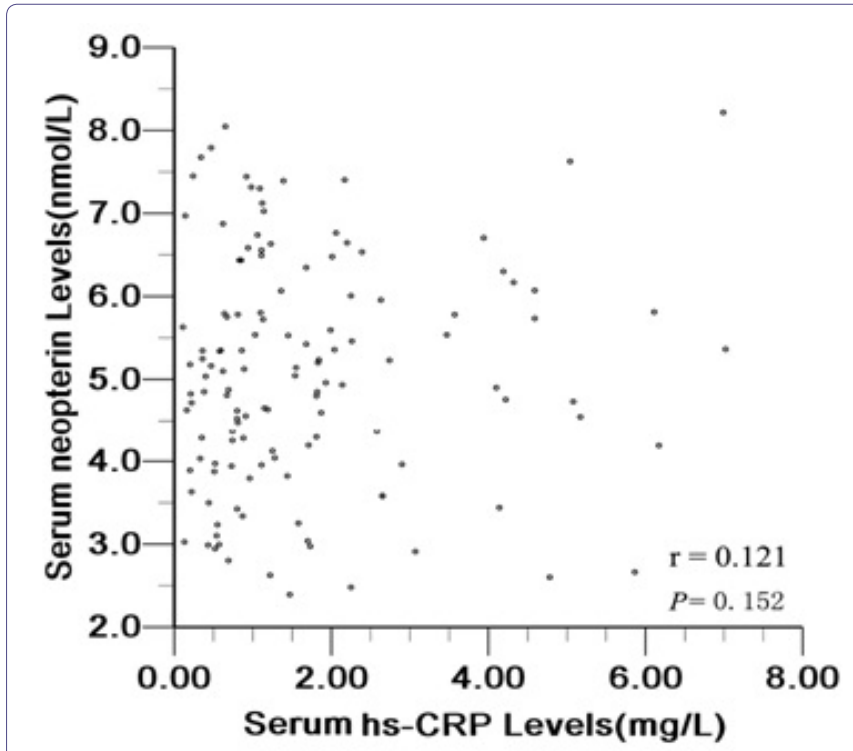

Figure 3: Correlation between serum neopterin and hs-CRP levels.

\begin{tabular}{|c|c|c|}
\hline & $\begin{array}{c}\text { Multivariate adjusted } \\
\text { OR (95 \% Cl) }\end{array}$ & P \\
\hline Age, per 6 increase $^{*}$ & $1.72(1.16-2.55)$ & $<0.05$ \\
\hline Hypertension (yes vs no) $^{*}$ & $0.72(0.26-2.00)$ & 0.53 \\
\hline WHR, per 0.06 increase $^{*}$ & $1.31(0.76-2.26)$ & 0.33 \\
\hline BMI, per $3 \mathrm{~kg} / \mathrm{m}^{2}$ increase $^{*}$ & $1.20(0.72-1.99)$ & 0.49 \\
\hline SBP, per $20 \mathrm{mmHg}$ increase & $1.56(0.93-2.61)$ & 0.09 \\
\hline HDL-C, per $0.3 \mathrm{mmol} /$ decrease $^{*}$ & $0.95(0.63-1.43)$ & 0.8 \\
\hline neopterin, per $1.0 \mathrm{nmol} /$ increase $^{\mathbf{4}}$ & $1.84(1.25-2.71)$ & $<0.05$ \\
\hline
\end{tabular}

Table 4: Binary logistic regression analysis for the prediction of CAS.

*Approximately $1 \mathrm{SD}$

$\triangle$ Approximately 1 IQR

\section{ROC curve}

Figure 4, shows the ROC curve of serum neopterin concentrations for the diagnosis of CAS. The AUC was $0.665(\mathrm{P}<0.01)$. The serum neopterin cutoff value of $5.1 \mathrm{nmol} / \mathrm{L}$ had a sensitivity of $68.6 \%$ and specificity of $61.5 \%$ in detecting CAS.

\section{Discussion}

In this community-base study with large sample, we measured circulating serum neopterin levels and investigating the cross-sectional relationship between serum neopterin levels and CAS assessed by ultrasound. Community residents without cardiovascular or cerebrovascular disease were enrolled. Subjects with the diseases which corroboratively or potentially affect the immune system were excluded. Such results were reliable.

Interestingly, some traditional risk factors such as age, WHR, BMI, history of hypertension, SBP, HDL-C were relevant with CAS in our study, which was in accordance with the results of the tromso study [20], though these risk factors were not statistically significant after multivariate adjustment in multivariate analysis except of age. Neopterin level remains an independent factor of CAS. The risk of CAS increased by 1.84 times with per $1.0 \mathrm{nmol} / \mathrm{L}$ (approximately 1 IQR) increased. Our results, in addition to the previous study, 


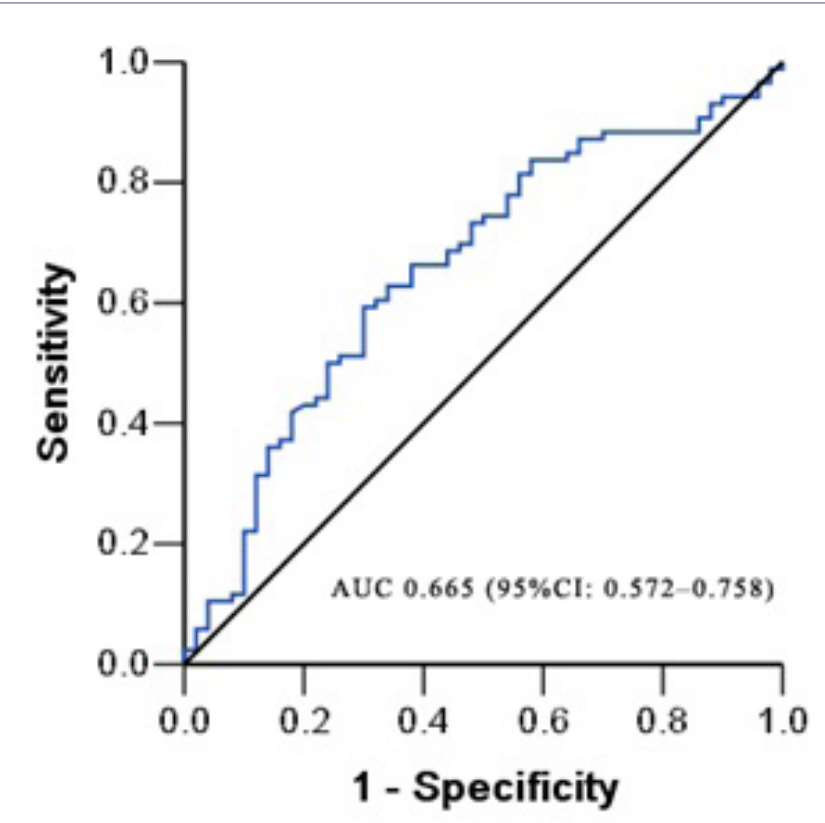

Figure 4: ROC curves of the serum neopterin level for the prediction of CAS.

demonstrate that circulating neopterin levels is increased in community population with subclinical carotid atherosclerosis. The results suggested involvement of immune system and the monocyte/ macrophage cell activation in the development of CAS.

Ultrasound is the one of the most common techniques used to evaluate CAS [21]. Carotid ultrasound examination is relatively inexpensive, widely available, portable and noninvasive [21]. In addition to imaging, biochemical findings have important clinical significance [22]. Occurrence and development of CAS are not simply lipid accumulation in the arterial wall but also a process of inflammation reaction [23]. During this process monocyte/macrophage cells occupy vital roles in vascular remodeling [24]. Pro-inflammatory cytokines released by Th1 cells and macrophages are major pro-atherogenic molecules [25]. Inflammatory cytokines involved in vascular inflammation stimulate the generation of endothelial adhesion molecules, proteases, and other mediators, which may enter the circulation in soluble form [25]. The concept of the involvement of inflammation in atherosclerosis has validated the adoption of inflammatory biomarkers for cerebrovascular risk stratification and prediction [26].

There have been many studies showing that neopterin may play an important role in the pathogenesis of atherosclerosis and represent also a marker for cardiovascular risk and cerebrovascular disease [27]. Patients with acute ischemic stroke had significantly higher serum levels of neopterin than controls without ischemic stroke [28]. The level was even higher at 1-year follow-up than at the acute stage, indicating chronic inflammatory activity and continuous macrophage activation in ischemic cerebrovascular diseases [29]. In recent years, several studies had a proved a significant correlation between neopterin levels and carotid IMT in different populations [30], which implied an association with the severity of carotid atherosclerosis. In accordance to the previous studies [31], the presence of complex carotid plaques detected by carotid ultrasound was related to increased circulating levels of neopterin. These findings suggest that neopterin is an important biomarker of plaque instability in carotid atherosclerotic lesions. However, the stenosis in the ICA is currently the benchmark criterion to determine how such disease is to be managed [32]. Research into plaque characteristics and associated inflammation may modify future selection criteria, but, currently, the degree of stenosis remains the cornerstone of management. In this study, we demonstrated that neopterin is associated with CAS and remain an independent risk factor when other confounding factors were adjusted. hs-CRP is a clinically valuable marker both from atherogenic and infectious point of view. However, in our study, there was neither significant difference in hs-CRP level between the CAS and control groups nor significant correlation between serum neopterin and hs-CRP levels.

This study still provided useful information reinforcing the key roles of inflammatory response in the pathogenesis of CAS and suggested a potential clinical use of neopterin as a serological biochemical marker which served as an alternate approach combining imaging studies in identifying an inflammatory CAS state in subclinical carotid atherosclerosis. Further clinical investigations confirming the direct association between serum neopterin levels and future risk of cerebrovascular events in a manner of cohort study, as well as discussing whether neopterin can be conductive for the decision making of individualized treatment programs such as anti-inflammatory therapy, or can be a parameter in monitoring the substantial benefit during therapeutic follow-up, are warranted.

However, there are still some drawbacks in this study addressed further below. First, the authors should further categorize the subjects based on the severity of CAS. But in our study, the participants were community subclinical subjects, mostly had mild stenosis, only 7 subjects were moderate stenosis. For this reason, it is unrealistic to further categorize the patients based on the severity of CAS. Even though a flaw it was, our study revealed that vascular inflammatory reaction has been prominent even when the carotid artery is in the primary stage with mild stenosis. Moreover, the authors did not study all of the subjects. However, random sampling was used in the study, so the sample is representative.

\section{Conclusion}

Our findings demonstrate that circulating neopterin level is increased in subclinical carotid atherosclerosis population with CAS and reinforce the key roles of inflammatory response in the pathogenesis.

\section{Acknowledgement}

Grant support: The present study was partially supported by National Natural Sciences Foundation of China Grant (30971028), Guangdong Province Natural Sciences Foundation (06021232), Guangdong Province Technological Grant (2007B031502003), Guangzhou City Technological Grant (2009ZL-E021) and the First Affiliated Hospital of Sun Yat-Sen Outstanding Young Talent Supporting Projects (18700112).

\section{References}

1. Mukherjee D, Patil CG (2011) Epidemiology and the global burden of stroke. World Neurosurg 76: 85-90.

2. Mathiesen EB, Johnsen SH (2009) Ultrasonographic measurements of subclinical carotid atherosclerosis in prediction of ischemic stroke. Acta Neurol Scand Suppl 189: 68-72.

3. White H, Boden-Albala B, Wang C, Elkind MS, Rundek T, et al. (2005) Ischemic stroke subtype incidence among whites, blacks, and Hispanics: the Northern Manhattan Study. Circulation 111: 1327-1331. 
4. Petty GW, Brown RD Jr, Whisnant JP, Sicks JD, O'Fallon WM, et al. (1999) Ischemic stroke subtypes: a population-based study of incidence and risk factors. Stroke 30: 2513-2516.

5. Grubb RL Jr, Derdeyn CP, Fritsch SM, Carpenter DA, Yundt KD, et al. (1998) Importance of hemodynamic factors in the prognosis of symptomatic carotid occlusion. JAMA 280: 1055-1060.

6. Tsiskaridze A, Devuyst G, de Freitas GR, van Melle G, Bogousslavsky $J$ (2001) Stroke with internal carotid artery stenosis. Arch Neurol 58: 605-609.

7. Ross R (1999) Atherosclerosis--an inflammatory disease. N Engl J Med 340: $115-126$.

8. Sabeti S, Exner M, Mlekusch W, Amighi J, Quehenberger P, et al. (2005) Prognostic impact of fibrinogen in carotid atherosclerosis: nonspecific indicator of inflammation or independent predictor of disease progression? Stroke 36: 1400-1404.

9. Alvarez Garcia B, Ruiz C, Chacon P, Sabin JA, Matas M (2003) High-sensitivity $\mathrm{C}$-reactive protein in high-grade carotid stenosis: risk marker for unstable carotid plaque. J Vasc Surg 38: 1018-1024.

10. Packard RR, Libby $P$ (2008) Inflammation in atherosclerosis: from vascular biology to biomarker discovery and risk prediction. Clin Chem 54: 24-38.

11. Huber C, Batchelor JR, Fuchs D, Hausen A, Lang A, et al. (1984) Immune response-associated production of neopterin. Release from macrophages primarily under control of interferon-gamma. J Exp Med 160: 310-316.

12. Murr C, Widner B, Wirleitner B, Fuchs D (2002) Neopterin as a marker for immune system activation. Curr Drug Metab 3: 175-187.

13. Schumacher M, Halwachs G, Tatzber F, Fruhwald FM, Zweiker R, et al. (1997) Increased neopterin in patients with chronic and acute coronary syndromes. J Am Coll Cardiol 30: 703-707.

14. Garcia-Moll X, Cole D, Zouridakis E, Kaski JC (2000) Increased serum neopterin: a marker of coronary artery disease activity in women. Heart 83: 346 350

15. Zierler R, Strandness D (1987) Noninvasive dynamic and real-time assessment of extracranial cerebrovasculature. In: Wood JH (ed.). Cerebral blood flow: Physiologic and clinical aspects. McGraw-Hill, New York, USA. Pg no: 311-323.

16. Chambless LE, Folsom AR, Clegg LX, Sharrett AR, Shahar E, et al. (2000) Carotid wall thickness is predictive of incident clinical stroke: the Atherosclerosis Risk in Communities (ARIC) study. Am J Epidemiol 151: 478-487.

17. Ebrahim S, Papacosta O, Whincup P, Wannamethee G, Walker M, et al. (1999) Carotid plaque, intima media thickness, cardiovascular risk factors and prevalent cardiovascular disease in men and women: the British Regional Heart Study. Stroke 30: 841-850.
18. Schulte-Altedorneburg G, Droste DW, Haas N, Kemény V, Nabavi DG, et al (2000) Preoperative B-mode ultrasound plaque appearance compared with carotid endarterectomy specimen histology. Acta Neurol Scand 101: 188194.

19. Knox RA, Breslau PJ, Strandness DE Jr (1982) A simple parameter for accurate detection of severe carotid disease. $\mathrm{Br} \mathrm{J}$ Surg 69: 230-233.

20. Joakimsen O, Bonaa KH, Stensland-Bugge E, Jacobsen BK (1999) Age and sex differences in the distribution and ultrasound morphology of carotid atherosclerosis: the Troms $\varnothing$ Study. Arterioscler Thromb Vasc Biol 19: 30073013.

21. Makris GC, Lavida A, Griffin M, Geroulakos G, Nicolaides AN (2011) Three-dimensional ultrasound imaging for the evaluation of carotid atherosclerosis. Atherosclerosis 219: 377-383.

22. Hermus L, Lefrandt JD, Tio RA, Breek JC, Zeebregts CJ (2010) Carotid plaque formation and serum biomarkers. Atherosclerosis 213: 21-29.

23. Baldassarre D, De Jong A, Amato M, Werba JP, Castelnuovo S, et al. (2008) Carotid intima-media thickness and markers of inflammation, endothelial damage and hemostasis. Ann Med 40: 21-44.

24. Boyle JJ (2005) Macrophage activation in atherosclerosis: pathogenesis and pharmacology of plaque rupture. Curr Vasc Pharmacol 3: 63-68.

25. Hansson GK, Robertson AK, Söderberg-Nauclér C (2006) Inflammation and atherosclerosis. Annu Rev Pathol 1: 297-329.

26. Packard RR, Libby $P$ (2008) Inflammation in atherosclerosis: from vascular biology to biomarker discovery and risk prediction. Clin Chem 54: 24-38.

27. Fuchs D, Avanzas P, Arroyo-Espliguero R, Jenny M, Consuegra-Sanchez L, et al. (2009) The role of neopterin in atherogenesis and cardiovascular risk assessment. Curr Med Chem 16: 4644-4653.

28. Cojocaru IM, Cojocaru M, Burcin C, Atanasiu A (2007) Detection of neopterin as parameter of potential monocyte activation in patients with acute ischemic stroke. Rom J Intern Med 45: 365-369.

29. Anwaar I, Gottsäter A, Lindgärde F, Mattiasson I (1999) Increasing plasma neopterin and persistent plasma endothelin during follow-up after acute cerebral ischemia. Angiology 50: 1-8.

30. Erten Y, Oztürk MA, Oktar S, Pasaoglu H, Reis KA, et al. (2005) Association between neopterin and carotid intima-media thickness in hemodialysis patients. Nephron Clin Pract 101: 134-138.

31. Sugioka K, Naruko T, Matsumura Y, Shirai N, Hozumi T, et al. (2010) Neopterin and atherosclerotic plaque instability in coronary and carotid arteries. J Atheroscler Thromb 17: 1115-1121.

32. Gray C, Murray SA, Connolly M, O'Donohoe MK, McDonnell CO, et al. (2015) The implications of different criteria for grading carotid artery stenosis by duplex ultrasound. J Clin Ultrasound 43: 563-566. 